\title{
Molecular orientation behavior of isotactic polypropylene under uniaxial stretching by rheo-Raman spectroscopy
}

\author{
T. Kida, Y. Hiejima*, K.-H. Nitta \\ Department of Chemical and Materials Science, Kanazawa University, Kakuma Campus, 920-1192 Kanazawa, Japan
}

Received 8 January 2016; accepted in revised form 12 March 2016

\begin{abstract}
The molecular orientation behavior of isotactic polypropylene (iPP) is investigated by using in situ Raman spectroscopy under tensile tests. A versatile method of the tilt-angle correction for the orientation parameters is newly developed, where the molecular orientation in highly oriented specimens is assumed to be entropically favorable. The real-time changes of orientation parameters and orientation distribution functions are determined for the molecular chain axis of iPP during uniaxial stretching. The molecular orientation remains random in the elastic region, and increases after the first yield point. In the yielding region, a broad distribution of orientation toward an intermediate angle of $30-70^{\circ}$ from the stretching direction is observed. This is interpreted as reorientation of the crystalline chains being hindered by rigid, bulky lamellar cluster units. After the yielding region, orientation toward the stretching direction proceeds rapidly, approaching highly oriented states.
\end{abstract}

Keywords: mechanical properties, Raman spectroscopy, molecular orientation, isotactic-polypropylene

\section{Introduction}

Isotactic polypropylene (iPP) is a semi-crystalline polymer which exhibits high strength, workability and transparency and has low cost. iPP is commonly used in films, plastic moldings and structural materials. The molecular orientation of the crystalline and amorphous chains greatly influences the mechanical properties of such semi-crystalline polymers $[1,2]$. For example, a high degree of orientation along the stretching axis is required for fabricating very strong fibers.

The molecular orientation of semi-crystalline polymers has been investigated by various experimental methods. Birefringence is a simple and fundamental technique which gives a qualitative measure of the overall orientation in bulk specimens, including the crystalline and amorphous regions [3, 4]. Wide-angle $\mathrm{X}$-ray diffraction (WAXD) basically provides the distribution of molecular orientation of the crystalline chain, while that of the amorphous chains are also determined by the synchrotron WAXD measurements [5, 6]. Infrared (IR) spectroscopy can separately evaluate the average molecular orientations of both crystalline and amorphous chains [7, 8], using its dichroic ratio. However, IR spectroscopy is unsuitable for thick samples such as plastic molds and structural materials, then, it has been applied for the sliced samples, because of high extinction coefficients in the IR region.

Recent improvements in laser light sources, optical devices and detectors have promoted the application of Raman spectroscopy across various research fields. Raman spectroscopy is a vibrational spectroscopy technique with several advantages over the abovementioned techniques. First, its sufficiently short acquisition time enables measurements to be carried out in situ [9-11]. The stress relaxation upon unloading polymeric samples can result in appreciable structural changes, including molecular orientation. Rheo-Raman spectroscopy which is the simultane- 
ous measurement of Raman spectra and mechanical tests enables real-time monitoring of the microscopic deformation mechanism. Second, polarized Raman spectroscopy detects the molecular orientations of both crystalline and amorphous chains. Raman spectroscopy has been used to evaluate the molecular orientations of crystalline and amorphous chains in iPP films [12, 13], and in situ micro-Raman spectroscopy has also been applied to investigate the microscopic mechanism of iPP during uniaxial deformation [9]. Third, the distribution of molecular orientations is determined by calculating the orientation distribution function (ODF) from the orientation parameters $\left\langle P_{2}\right\rangle$ and $\left\langle P_{4}\right\rangle[14,15]$. In contrast, IR spectroscopy can only provide the orientation function as a measure of the average orientation. Finally, since typical optical setups for the scattering measurements are more flexible than those for the transmission measurements, Raman spectroscopy can provide noncontact and non-destructive technique to probe the molecular orientation of plastic molds without any pretreatments.

The crystalline chain of iPP has a 3 helical structure [16], so the principal axis of the Raman tensor is not parallel to the molecular chain axis [13, 17]. A correction for the tilt angle between these two axes is required to evaluate the orientation of the molecular chains. Tanaka and Young reported tilt-angle corrections using WAXD measurements [13, 18], and obtained ODF values using the $1000 \mathrm{~cm}^{-1}$ band of highly drawn iPP. The ODF values of poly(lactic acid), which also forms helical chains in the crystalline phase, have been determined from highly drawn samples [13, 18, 19].

In the current study, rheo-Raman spectroscopy is used to elucidate the molecular orientation behavior of iPP during elongation. A new method for correcting the tilt angle between the molecular axis and the principal axis of the Raman tensor is applied. ODF values and orientation parameters for the crystalline chains of iPP are obtained. The microscopic mechanism of deformation of iPP under uniaxial tensile testing is discussed, by comparing the current results with previous results for high-density polyethylene (HDPE) $[10,11]$.

\section{Methods}

\subsection{Sample and apparatus}

Ziegler-Natta-catalyzed homo iPP pellets $\left(M_{\mathrm{w}}=\right.$ $\left.4.9 \cdot 10^{5}, M_{\mathrm{w}} / M_{\mathrm{n}}=4.4\right)$ supplied by Union Polymer Material Co., Ltd (Dalian, China, Grade: YD101-A) were used in this study. The pellets were compression molded in a hot press at $230^{\circ} \mathrm{C}$ and $20 \mathrm{MPa}$ for $5 \mathrm{~min}$, to prepare a sheet with thickness of about $1 \mathrm{~mm}$. The sample sheet was removed from the hot press, quenched in boiled water at $100^{\circ} \mathrm{C}$. The density of the sample was determined to be $908 \mathrm{~kg} \mathrm{~m}^{-3}$ by the Archimedes method. Its volumetric crystallinity was determined to be $66 \%$, where the densities of the amorphous and crystalline regions were assumed to be $\rho_{\mathrm{a}}=854$ and $\rho_{\mathrm{c}}=936 \mathrm{~kg} \mathrm{~m}^{-3}$, respectively [20]. Test specimens for tensile tests were cut out of the sample sheet with a notch-shaped die ( $2 \mathrm{~mm}$ gauge length, $4 \mathrm{~mm}$ width in Figure 1).

The rheo-Raman spectroscopy apparatus is shown schematically in Figure 2. A custom-made tensile tester with a double-drawing mechanism was installed in the Raman spectroscopic apparatus, the details of which are reported elsewhere [10, 11]. Laser light from a DPSS laser (LASOS, Jena, Germany, $637.9 \mathrm{~nm}$, $200 \mathrm{~mW}$ ) was monochromated with a laser line filter, and irradiated into the notched portion of the specimen, with a spot size of $1 \mathrm{~mm}$ in diameter. The elongation speed was set to $1 \mathrm{~mm} / \mathrm{min}$, and the stretching temperature was $20^{\circ} \mathrm{C}$. The scattered light was collected with a pair of convex lenses, where the excitation light was removed with a Raman long-pass filter. A charge-coupled device camera equipped with a monochromator (PIXIS100 and SpectraPro 2300i, Princeton Instruments, Trenton, USA, NJ) was used as the detector. For polarized Raman spectroscopy, a pair of wire-grid polarizers was inserted as the polarizer and

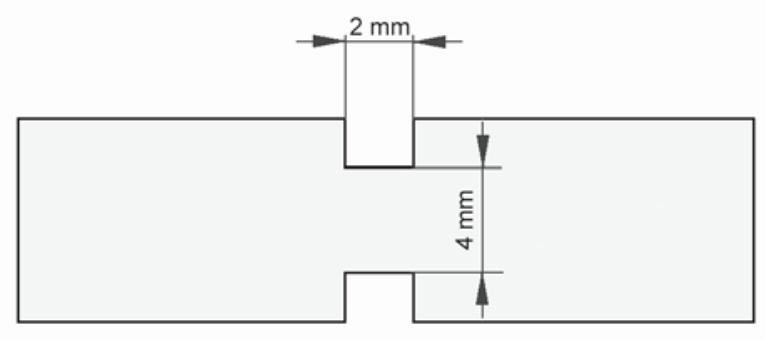

Figure 1. Schematic illustraion of sample specimen 


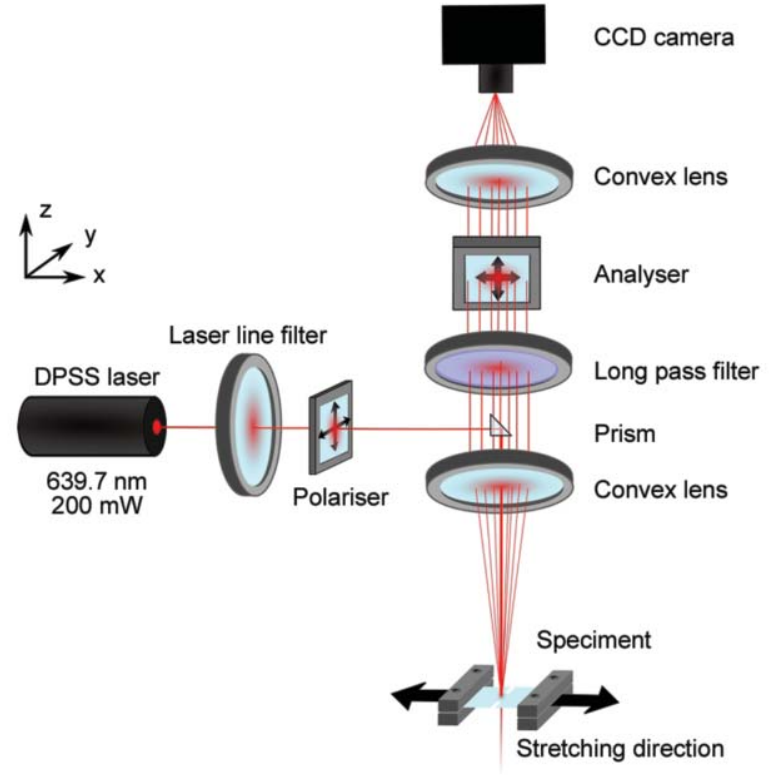

Figure 2. Schematic illustration of rheo-Raman spectroscopic apparatus

analyzer. The polarized spectra were collected in the $z z, y z$, and $y y$ geometries and accumulated 10 times with an exposure time of $500 \mathrm{~ms}$. It is noteworthy that the total exposure time of $5 \mathrm{~s}$ can be shortened up to $\sim 1 \mathrm{~s}$, with maintaining sufficient signal to noise ratio.

\subsection{In situ Raman spectroscopy}

Typical in situ polarized Raman spectra are shown in Figure 3. Assignments of the Raman bands of iPP are listed in Table $1[13,21]$. The Raman spectra of undrawn specimens were independent of polarization, indicating that the molecular orientation was random. After elongation, the anti-symmetric $\left(975 \mathrm{~cm}^{-1}\right)$ and symmetric $\left(809 \mathrm{~cm}^{-1}\right)$ stretching modes were
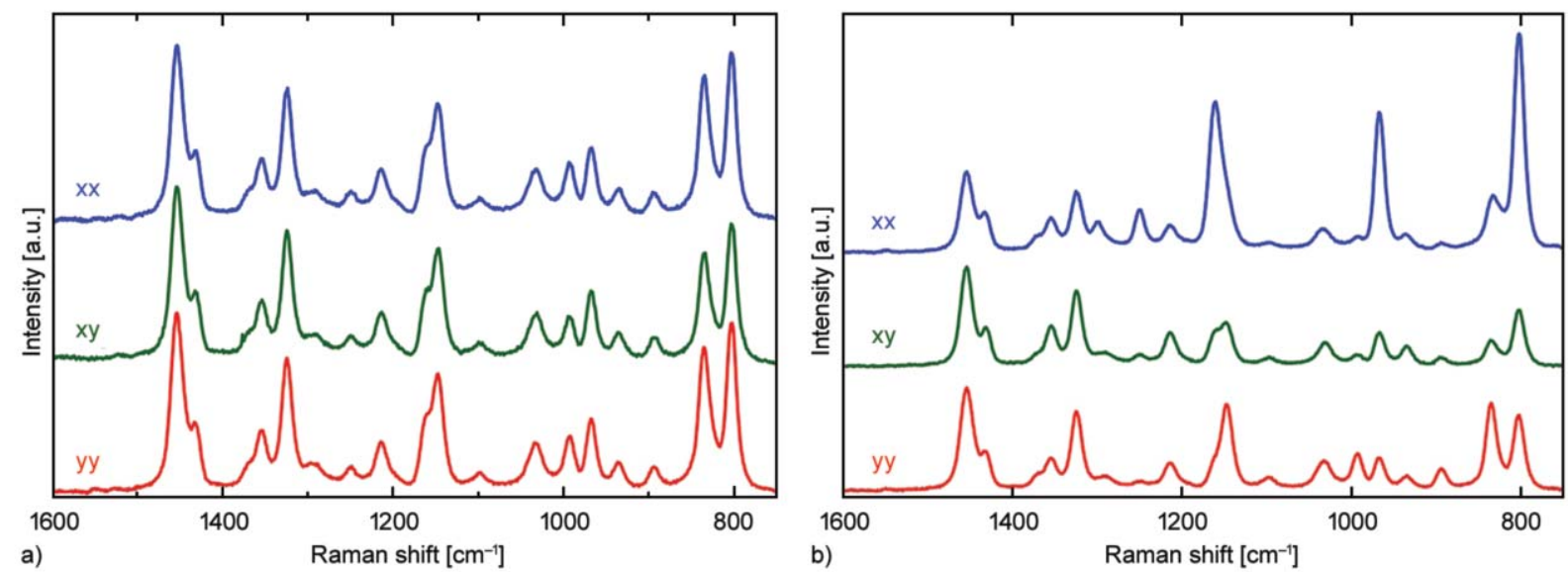

Figure 3. In situ polarized Raman spectra of iPP at (a) $\varepsilon=0$ and (b) $\varepsilon=8$. The polarization conditions are attached to each spectrum

Table 1. Vibrational and phase assignments for the Raman spectrum of iPP $[13,21]$

\begin{tabular}{|c|c|c|c|}
\hline $\begin{array}{c}\text { Peak position } \\
{\left[\mathbf{c m}^{-1}\right]}\end{array}$ & $\begin{array}{c}\text { Vibrational } \\
\text { mode }^{*}\end{array}$ & Phase & $\begin{array}{c}\text { Raman } \\
\text { tensor form }\end{array}$ \\
\hline 809 & $v_{\mathrm{s}}(\mathrm{C}-\mathrm{C})+r\left(\mathrm{CH}_{2}\right)$ & Crystalline & $\mathrm{A}$ \\
\hline 830 & $v(\mathrm{C}-\mathrm{C})$ & Amorphous & $\mathrm{A}$ \\
\hline 840 & $r\left(\mathrm{CH}_{2}\right)$ & $\begin{array}{c}\text { Crystalline }+ \\
\text { Amorphous }\end{array}$ & $\mathrm{A}$ \\
\hline 975 & $v_{\text {as }}(\mathrm{C}-\mathrm{C})+r\left(\mathrm{CH}_{3}\right)$ & Crystalline & $\mathrm{A}$ \\
\hline
\end{tabular}

${ }^{*} v$ : stretching vibration (as: anti-symmetric, $s$ : symmetric), $r$ : rocking vibration

strongly dependent on the polarization conditions, suggesting that highly oriented states were attained. The Raman spectra were fitted with a sum of Voigt functions using a nonlinear Levenberg-Marquardt method, and the areas for the two peaks were determined. The errors of the peak areas in the curve fitting were less than $\pm 2.0 \%$ for the $809 \mathrm{~cm}^{-1}$ band, and $\pm 4.0 \%$ for the $975 \mathrm{~cm}^{-1}$ band.

\subsection{Orientation parameters}

The orientation parameters $\left\langle P_{2}\right\rangle$ and $\left\langle P_{4}\right\rangle$ which represented the molecular orientation were defined as given by Equations (1) and (2):

$\left\langle P_{2}\right\rangle=\frac{3\left\langle\cos ^{2} \theta\right\rangle-1}{2}$

$\left\langle P_{4}\right\rangle=\frac{35\left\langle\cos ^{4} \theta\right\rangle-30\left\langle\cos ^{2} \theta\right\rangle+3}{8}$

where $\theta$ denotes the angle between the principal axis of the Raman tensor and the stretching axis. The orientation parameters were obtained from the intensities of the polarized Raman spectra as defined by Equations (3)-(6) [15, 19, 22-24]:

b) 


$$
\begin{aligned}
& I_{y y}=b\left(\frac{8 a^{2}+4 a+3}{15}+2\left\langle P_{2}\right\rangle \frac{4 a^{2}-a-3}{21}+3\left\langle P_{4}\right\rangle \frac{a^{2}-2 a+1}{21}\right) \\
& I_{x x}=b\left(\frac{8 a^{2}+4 a+3}{15}-4\left\langle P_{2}\right\rangle \frac{4 a^{2}-a-3}{21}+8\left\langle P_{4}\right\rangle \frac{a^{2}-2 a+1}{35}\right) \\
& I_{x y}=b\left(\frac{a^{2}-2 a+1}{15}+\left\langle P_{2}\right\rangle \frac{a^{2}-2 a+1}{21}-4\left\langle P_{4}\right\rangle \frac{a^{2}-2 a+1}{35}\right)
\end{aligned}
$$

with

$$
\frac{I_{x y}}{I_{x x}}=\frac{a^{2}-2 a+1}{8 a^{2}+4 a+3}
$$

where $I_{\mathrm{ij}}$ denotes the integrated intensity for the $i$-polarized excitation and $j$-polarized scattered light. The terms $a$ and $b$ were associated with the principal elements of the Raman tensor [15, 22].

The crystalline chain of iPP has $\mathrm{C}_{3}$ symmetry, because of its $3_{1}$ helical conformation. Thus, the forms of the Raman tensor were classified mainly as type A or E [15]. Raman bands classified as type A can be used to calculate the orientation parameters [13, 22-24], so the Raman bands at 809 and $975 \mathrm{~cm}^{-1}$ were used. Both of these are classified as type A, and were sufficiently strong to calculate the orientation parameters.

\subsection{Tilt-angle correction}

The orientation parameters obtained by solving Equations (3)-(6) provided information on the orientation of the principal axis of the Raman tensor, but not on the orientation of the molecular chain axis. Given that $3_{1}$ helical chains were formed in the crystalline phase of iPP, a correction for the tilt angle between the principal and molecular axes was required $[13,17,19]$. The $l$-th orientation parameters $\left\langle P_{1}^{\text {chain }}\right\rangle$ corrected for the tilt angle $\beta$ can be written as shown in Equation (7) $[13,17]$ :

$$
\left\langle P_{1}^{\text {chain }}\right\rangle=\frac{\left\langle P_{1}\right\rangle}{P_{1}(\cos \beta)}
$$

where $P_{1}(x)$ is the $l$-th Legendre polynomial.

The values of the tilt-angle $\beta$ of several Raman bands of iPP had been determined by using the results of the WAXD experiments [13]. They had successfully estimated the value of $\left\langle P_{2}{ }^{\text {chain }}\right\rangle$ for drawn specimens of iPP for the Raman band at $998 \mathrm{~cm}^{-1}$, though they had failed to use the Raman band at 810 and $1221 \mathrm{~cm}^{-1}$ [13]. To determine the value of $\beta$ solely from the Raman spectra in a self-consistent manner, we assumed that the molecular orientation at high extension- al ratio was described by an ideal uniaxial orientation around the stretching direction; $\left\langle P_{4}{ }^{\text {chain }}\right\rangle$ and $P_{4}(\cos \beta)$ were assumed to be replaced by their entropically favorable values $\left\langle P_{4}{ }^{\text {chain }}\right\rangle_{\mathrm{mp}}$ and $P_{4, \mathrm{mp}}(\cos \beta)$, respectively $[11,24]$. In fact, the values of $\left\langle P_{4}\right.$ chain $\rangle$ at high extensional ratios are very close to that of $\left\langle P_{4}{ }^{\text {chain }}\right\rangle_{\text {mp }}$ for HDPE [11, 24], iPP [13], and poly(ethylene terephthalate) [24]. Then, at high extensional rates, Equation (7) for $l=2$ and 4 can be written as Equations (8) and (9):

$$
\begin{aligned}
\left\langle P_{2}^{\text {chain }}\right\rangle & =\frac{\left\langle P_{2}\right\rangle}{P_{2}(\cos \beta)} \\
\left\langle P_{4}^{\text {chain }}\right\rangle & =\frac{\left\langle P_{4}\right\rangle}{P_{4, \text { mp }}(\cos \beta)}
\end{aligned}
$$

respectively. The most-probable values $\left\langle P_{4}\right\rangle_{\text {mp }}$ were determined to maximize the information entropy, and approximately written as the polynomials of $\left\langle P_{2}\right\rangle$ as Equations (10) and (11) [24, 25]:

$$
\begin{aligned}
\left\langle P_{4}\right\rangle_{\mathrm{mp}}=- & 0.083\left\langle P_{2}\right\rangle+1.366\left\langle P_{2}\right\rangle^{2}- \\
& 1.899\left\langle P_{2}\right\rangle^{3}+1.616\left\langle P_{2}\right\rangle^{4}
\end{aligned}
$$

for positive $\left\langle P_{2}\right\rangle$ values, and

$$
\begin{aligned}
\left\langle P_{4}\right\rangle_{\mathrm{mp}}=- & 0.052\left\langle P_{2}\right\rangle+1.574\left\langle P_{2}\right\rangle^{2}+ \\
& 3.968\left\langle P_{2}\right\rangle^{3}+8.058\left\langle P_{2}\right\rangle^{4}
\end{aligned}
$$

for negative $\left\langle P_{2}\right\rangle$ values. By combining Equations (8)-(11), the tilt angle $\beta$ was estimated from the experimental $\left\langle P_{2}\right\rangle$ and $\left\langle P_{4}\right\rangle$ at high extensional ratios. Thus, Equation (7) with the obtained value of $\beta$ gives the tilt-angle correction for the orientation parameters. It is noteworthy that the present method provides the corrected orientation parameters from a set of polarized Raman spectra of specimens under stretching with no auxiliary experiments.

\subsection{Orientation distribution function (ODF)}

The ODF $\mathrm{N}(\theta)$ which represented the angular distribution of the molecular orientation was defined asby Equation (12) [15, 25]: 
$N(\theta)=\sum_{1=0}^{\infty}\left(\frac{2 l+1}{2}\right)\left\langle P_{1}^{\text {chain }}\right\rangle P_{1}(\cos \theta)$

Although the polarized Raman spectrum gives only two orientation parameters $\left(\left\langle P_{2}{ }^{\text {chain }}\right\rangle\right.$ and $\left.\left\langle P_{4}{ }^{\text {chain }}\right\rangle\right)$, the orientation parameters at higher ranks can also be determined by maximizing the information entropy $[13,25]$. The most probable orientation distribution function was described asby Equation (13) [13, 25]:

$N(\theta)=\frac{\exp \left[\lambda_{2} P_{2}(\cos \theta)+\lambda_{4} P_{4}(\cos \theta)\right]}{\int_{0}^{\pi} \sin \theta \mathrm{d} \theta \exp \left[\lambda_{2} P_{2}(\cos \theta)+\lambda_{4} P_{4}(\cos \theta)\right]}$

where the Lagrange multipliers $\lambda_{2}$ and $\lambda_{4}$ are determined to satisfy the following two constraints, described by Equations (14) and (15) for the experimental values of $\left\langle P_{2}{ }^{\text {chain }}\right\rangle$ and $\left\langle P_{4}{ }^{\text {chain }}\right\rangle$ :

$$
\begin{aligned}
& \left\langle P_{2}^{\text {chain }}\right\rangle=\int_{0}^{\pi} \sin \theta \mathrm{d} \theta P_{2}(\cos \theta) N(\theta) \\
& \left\langle P_{4}^{\text {chain }}\right\rangle=\int_{0}^{\pi} \sin \theta \mathrm{d} \theta P_{4}(\cos \theta) N(\theta)
\end{aligned}
$$

\section{Results and discussion}

Figure 4 shows the strain dependences of the orientation parameters for the principal axis of the Raman tensors of the symmetric and anti-symmetric $\mathrm{C}-\mathrm{C}$ stretching modes. While the orientation parameters show similar strain dependence, the values for the $809 \mathrm{~cm}^{-1}$ band are substantially smaller than those for the $975 \mathrm{~cm}^{-1}$ band.

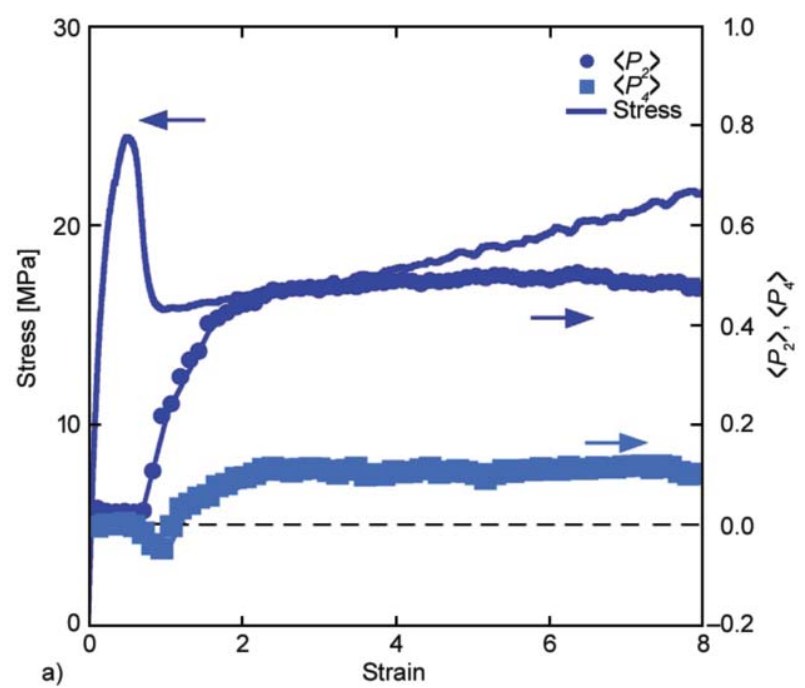

Table 2. Tilt angle $\beta$ calculated from Raman spectroscopy and WAXD data [13]

\begin{tabular}{|c|c|c|}
\hline $\begin{array}{c}\text { Raman band } \\
{\left[\mathbf{c m}^{-\mathbf{1}}\right]}\end{array}$ & $\boldsymbol{\beta}$ (Raman) & $\boldsymbol{\beta}$ (WAXD) \\
\hline 809 & 29.2 & $\sim 28.0$ \\
\hline 975 & 16.0 & 16.6 \\
\hline
\end{tabular}

In Table 2 , the tilt angles $\beta$ obtained by the tilt-angle correction with Equations (8) and (9) are compared with those previously determined by WAXD [13]. These values agree well with each other, suggesting that the present analysis can be safely applied for the tilt-angle correction of iPP.

In Figure 5, the orientation parameters for the molecular chain are obtained with the tilt-angle correction with Equation (7). The values of $\left\langle P_{2}{ }^{\text {chain }}\right\rangle$ and $\left\langle P_{4}{ }^{\text {chain }}\right\rangle$ for the Raman band at $809 \mathrm{~cm}^{-1}$ agree well with those for the $975 \mathrm{~cm}^{-1}$ band. This suggests that the apparent difference in the orientation parameters in Figure 4 is caused by the tilt angle. The slight difference in the orientation parameters between these two bands seems to result from uncertainty in the curve fitting. This is because fitting of the $809 \mathrm{~cm}^{-1}$ band is slightly affected by a nearby weak broad peak of the amorphous phase [9]. The consistency of the orientation parameters and the close agreement of tilt angles demonstrate that the present tilt angle correction gives $\left\langle P_{2}{ }^{\text {chain }}\right\rangle$ and $\left\langle P_{4}{ }^{\text {chain }}\right\rangle$ solely from a set of rheo-Raman spectra in a self-consistent method.

In the elastic region, $\left\langle P_{2}\right.$ chain $\rangle$ remains almost zero, indicating that the orientation of the crystalline chain remains random. While the macroscopic stress loaded

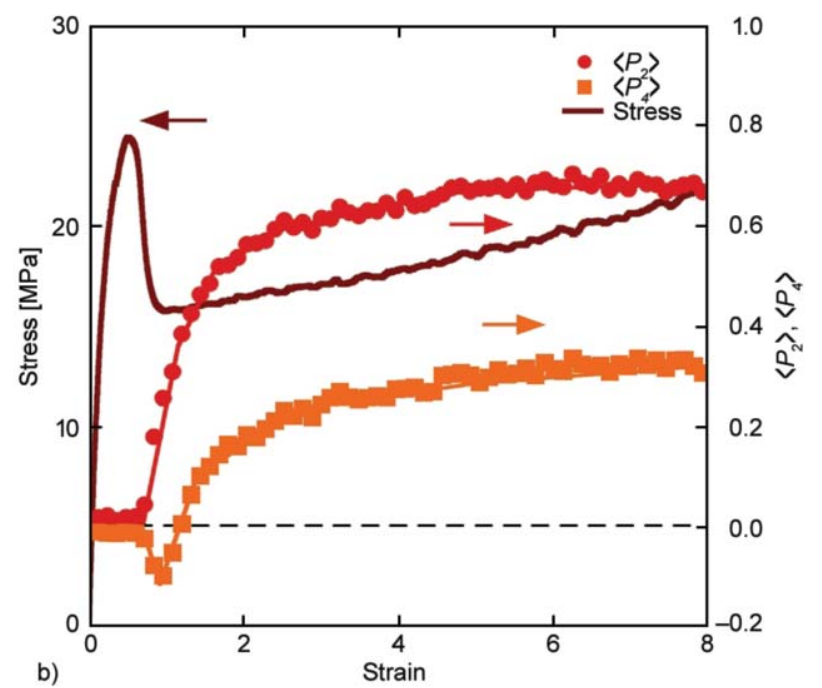

Figure 4. Stress-strain curves and strain dependences of the orientaion parameters for the principal axis of the Raman tensor of the Raman bands at (a) 809 and (b) $975 \mathrm{~cm}^{-1}$ 

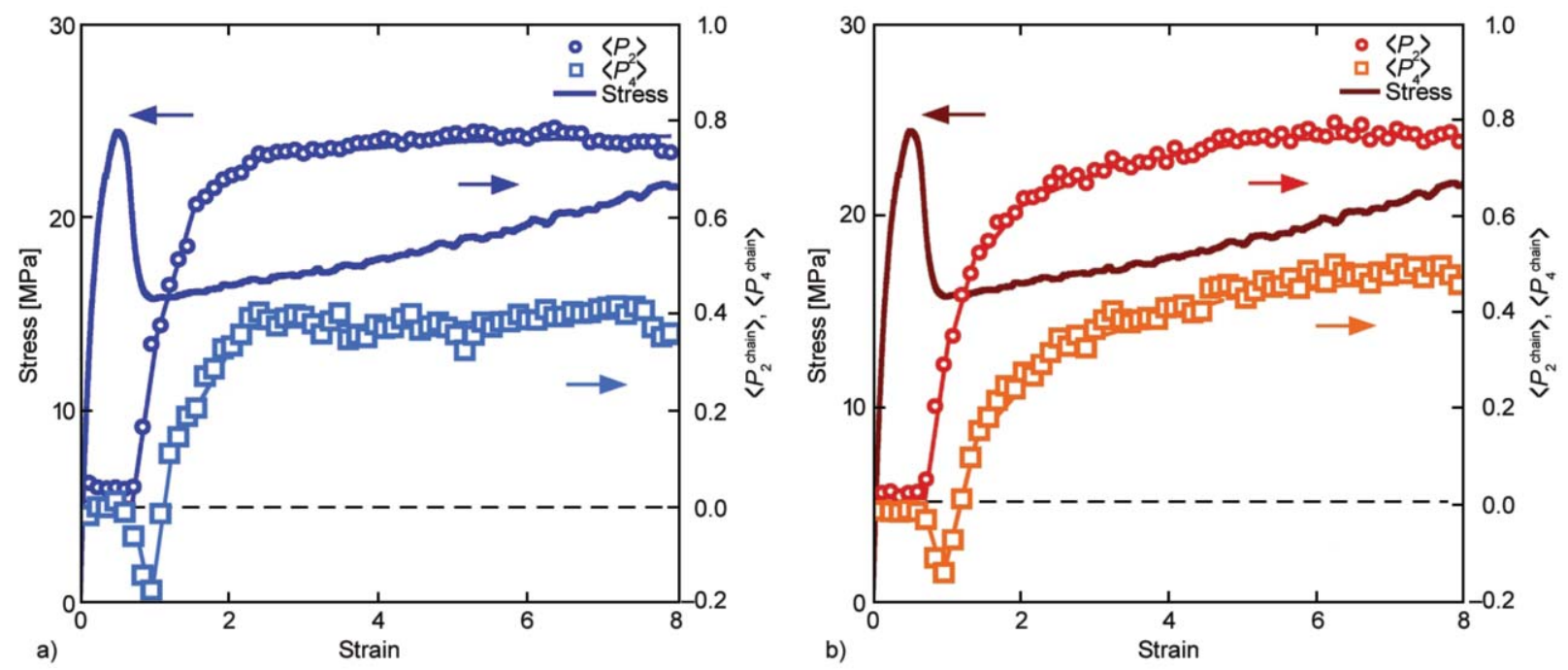

Figure 5. Stress-strain curves and strain dependences of the orientaion parameters for the molecular chain axis calculated from the Raman bands at (a) 809 and (b) $975 \mathrm{~cm}^{-1}$

on the specimen sharply increases with increasing strain, no orientational change is observed for the crystalline chains. This suggests that microscopic deformation takes place only in the amorphous phase. The amorphous phase in spherulites is reportedly mainly deformed in the elastic region [26, 27]. Recent in situ micro-SAXS measurements indicate that the thickness of the amorphous layers in spherulites is drastically changed; thickening and thinning of the amorphous layers in the polar and equatorial zones, respectively [28]. The inhomogeneous changes in the amorphous phase in spherulites have been assigned as the dominant mechanism during elastic deformation. $\left\langle P_{2}{ }^{\text {chain }}\right\rangle$ begins to increase after the first yield point (the maximum point of stress at around a strain of 0.5), and then sharply increases after the yielding region. This behavior of $\left\langle P_{2}{ }^{\text {chain }}\right\rangle$ indicates that the crystalline chains are oriented toward the stretching direction after yielding. In the strain-hardening region, $\left\langle P_{2}{ }^{\text {chain }}\right\rangle$ reaches an asymptotic value of $\sim 0.8$. The strain dependence of $\left\langle P_{2}{ }^{\text {chain }}\right\rangle$ under uniaxial stretching is in good agreement with previous results from in situ IR spectroscopy studies, [7, 29] and Raman spectroscopy results of drawn samples $[9,13]$. While the values of $\left\langle P_{4}{ }^{\text {chain }}\right\rangle$ remain constant in the elastic region, $\left\langle P_{4}{ }^{\text {chain }}\right\rangle$ decreases with increasing strain and exhibits a minimum in the yielding region. This behavior of $\left\langle P_{4}\right.$ chain $\rangle$ during yielding has also been observed for HDPE. [10, 11] After the yielding region, $\left\langle P_{4}{ }^{\text {chain }}\right\rangle$ shows similar strain dependence with that of $\left\langle P_{2}\right.$ chain $\rangle$. The values of $\left\langle P_{4}\right.$ chain $\rangle$ at high extensional ratios are also consistent with those of highly drawn iPP films and fibers $[13,30]$.

Figure 6 shows the ODF values calculated from the orientation parameters for the $975 \mathrm{~cm}^{-1}$ band. In the elastic region, the ODF is constant irrespective of the polar angle, indicating that the crystalline chain has a random orientation. In the yielding region, where $\left\langle P_{4}{ }^{\text {chain }}\right\rangle$ shows a minimum, the ODF shows a broad peak at an intermediate angle of $\theta=30-70^{\circ}$ from the stretching direction. This orientational behavior during yielding has also been observed for HDPE [10, 11, 5]. It has been interpreted as the orientation of crystalline chains being hindered by rigid and bulky stacked lamellae (lamellar cluster units) $[10,11,5]$. The interpretation of lamellar cluster units appears to also be valid for iPP, because these units have also been observed in iPP [31]. The peak of the ODF at

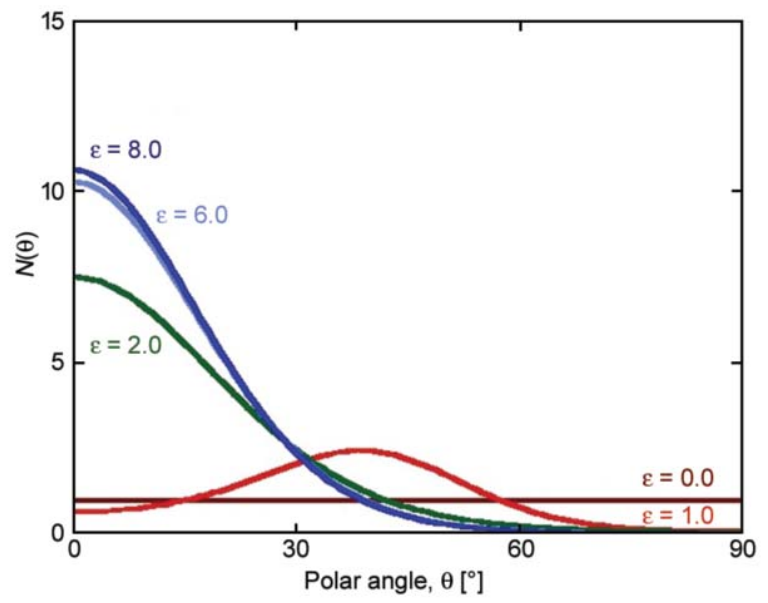

Figure 6. Orientation distribution functions of iPP at various strains 


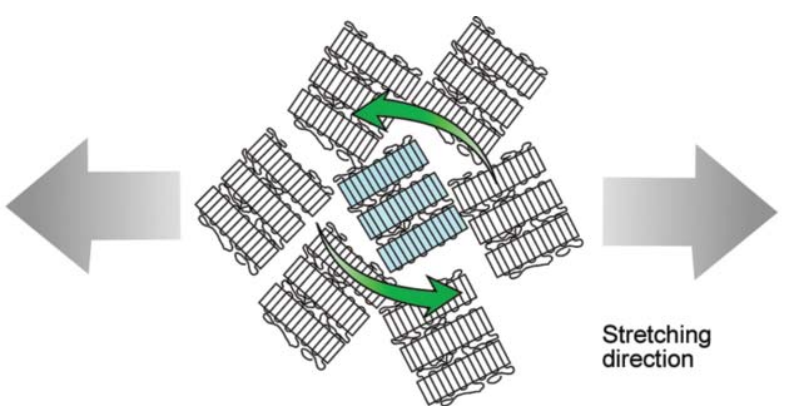

Figure 7. Schematic illustration of reorientation of the lamellar cluster units in the yielding region

$30-70^{\circ}$ for iPP in the yielding region is slightly shaper than that for HDPE, which may be explained by the size of their lamellar clusters (61 nm for iPP, $40 \mathrm{~nm}$ for HDPE) [11, 31, 32]. Reorientation of the lamellar cluster units is considered to be the dominant mechanism of molecular orientation in the yielding region, and the orientation of the crystalline chains toward the stretching direction is suppressed because one lamellar cluster units is surrounded by other cluster units as shown in Figure 7 [33]. Therefore, the reorientation of larger units for iPP is likely to be more hindered.

In the strain-hardening region, each ODF has a prominent peak at the stretching direction, and the ODF values above $\theta=60^{\circ}$ are practically zero. These observations indicate that the crystalline chains are oriented towards the stretching direction. The unimodal distribution around the stretching direction is consistent with those of drawn iPP films and rod samples determined from WAXD experiments [34, 35].

\section{Conclusions}

The molecular orientation of the crystalline chains of iPP under uniaxial deformation was investigated by rheo-Raman spectroscopy. A new method of tiltangle correction between the molecular chain axis and principal axis of the Raman tensor was proposed, and the real-time monitoring of the molecular orientation of iPP under stretching was performed. By using this method, the orientation parameters of the crystalline chain were calculated from the symmetric $\mathrm{C}-\mathrm{C}$ stretching mode at $809 \mathrm{~cm}^{-1}$, as well as the anti-symmetric $\mathrm{C}-\mathrm{C}$ stretching mode at $975 \mathrm{~cm}^{-1}$. The molecular orientation of the crystalline chains started after the first yield point, and proceeded rapidly in the yielding region. The ODF exhibited a broad peak in the yielding region, suggesting that molecular orien- tation was suppressed. This was attributed to the reorientation of bulky rigid lamellar cluster units being hindered by their large exclude volume. The assumption of entropically-favorable orientations for highlystretched specimens seems to be valid in many polymeric systems. Thus, rheo-Raman spectroscopy combined with the present tilt-angle correction can be widely utilized to elucidate orientational behavior at the molecular level. This methodology is currently being applied to other semi-crystalline polymers in our laboratory.

\section{Acknowledgements}

The authors thank Dr. Takashi Uneyama from Kanazawa University for providing the software to calculate ODF values. YH is thankful for financial support by JSPS KAKENHI (Grant Number 26410221).

\section{References}

[1] Ward I. M.: Structure and properties of oriented polymers. Chapman and Hall, London (1997).

[2] Ward I. M., Sweeney J.: Mechanical properties of solid polymers. Wiley, London (2013).

[3] Satija S. K., Wang C. H.: Polarized Raman scattering studies of chain orientation in hydrostatically extruded polypropylene. The Journal of Chemical Physics, 69, 2739-2744 (1978).

DOI: $10.1063 / 1.436870$

[4] Lefévre T., Pellerin C., Michel P.: Characterization of molecular orientation. in 'Comprehensive analytical chemistry' (eds.: Chalmers J. M., Meier R. J.) Elsevier, Oxford, Vol 53, 295-335 (2008).

[5] Jiang Z., Tang Y., Rieger J., Enderle H-F., Lilge D., Roth S. V., Gehrke R., Wu Z., Li Z., Men Y.: Structural evolution of tensile deformed high-density polyethylene at elevated temperatures: Scanning synchrotron small- and wide-angle X-ray scattering studies. Polymer, 50, 4101-4111 (2009).

DOI: 10.1016/j.polymer.2009.06.063

[6] Voyiatzis G., Petekidis G., Vlassopoulos D., Kamitsos E. I., Bruggeman A.: Molecular orientation in polyester films using polarized laser raman and fourier transform infrared spectroscopies and X-ray diffraction. Macromolecules, 29, 2244-2252 (1996).

DOI: $10.1021 / \mathrm{ma951199g}$

[7] Song Y., Nitta K-H., Nemoto N.: Deformation mechanisms of polymer thin films by simultaneous kinetic measurement of microscopic infrared dichroism and macroscopic stress. Part III: Influence of morphology on molecular orientation of isotactic polypropylene films subjected to uniaxial stretching. Nihon Reoroji Gakkaishi, 31, 131-141 (2003).

DOI: $10.1678 /$ rheology.31.131 
[8] Li H., Zhou W., Ji Y., Hong Z., Miao B., Li X., Zhang J., Qi Z., Wang X., Li L., Li Z-M.: Spatial distribution of crystal orientation in neck propagation: An in-situ microscopic infrared imaging study on polyethylene. Polymer, 54, 972-979 (2013).

DOI: $10.1016 /$ j.polymer.2012.12.012

[9] Martin J., Ponçot M., Hiver J. M., Bourson P., Dahoun A.: Real-time Raman spectroscopy measurements to study the uniaxial tension of isotactic polypropylene: A global overview of microstructural deformation mechanisms. Journal of Raman Spectroscopy, 44, 776-784 (2013).

DOI: $10.1002 / j \mathrm{js} .4244$

[10] Kida T., Hiejima Y. Nitta K-H.: Rheo-optical Raman study of microscopic deformation in high-density polyethylene under hot drawing. Polymer Testing, 44, 3036 (2015).

DOI: $10.1016 /$ j.polymertesting.2015.03.018

[11] Kida T., Oku T., Hiejima Y., Nitta K-H.: Deformation mechanism of high-density polyethylene probed by in situ Raman spectroscopy. Polymer, 58, 88-95 (2015). DOI: $10.1016 /$ j.polymer.2014.12.030

[12] Maxfield J., Stein R. S., Chen M. C.: Polarized Raman studies of crystalline and amorphous orientation in polyethylene. Journal of Polymer Science Polymer Physics Edition, 16, 37-48 (1978). DOI: $10.1002 /$ pol.1978.180160104

[13] Tanaka M., Young R. J.: Molecular orientation distributions in the crystalline and amorphous regions of uniaxially oriented isotactic polypropylene films determined by polarized Raman spectroscopy. Journal of Macromolecular Science Part B: Physics, 44, 967-991 (2005). DOI: 10.1080/00222340500323599

[14] Lapersonne P., Bower D. I., Ward I. M.: Molecular orientation and conformational changes due to uniaxialplanar deformation of poly(ethylene terephthalate) films. Polymer, 33, 1277-1283 (1992).

DOI: $10.1016 / 0032-3861(92) 90774-Q$

[15] Bower D. I.: Investigation of molecular orientation distributions by polarized Raman scattering and polarized fluorescence. Journal of Polymer Science Part B: Polymer Physics Edition, 10, 2135-2153 (1972).

DOI: $10.1002 /$ pol.1972.180101103

[16] Moore E. P. Jr.: Polypropylene handbook. Hanser, Cincinnati (1996).

[17] Lovell R., Mitchell G.: Molecular orientation distribution derived from an arbitrary reflection. Acta Crystallographica Section A, 37, 135-137 (1981).

DOI: $10.1107 / \mathrm{S} 0567739481000247$

[18] Tanaka M., Young R. J.: Molecular orientation distributions in uniaxially oriented poly(L-lactic acid) films determined by polarized Raman spectroscopy. Macromolecules, 39, 3312-3321 (2006).

DOI: $10.1021 / \mathrm{ma} 0526286$
[19] Tanaka M., Young R. J.: Review polarised Raman spectroscopy for the study of molecular orientation distributions in polymers. Journal of Materials Science, 41, 963-991 (2006). DOI: $10.1007 / \mathrm{s} 10853-006-6595-7$

[20] Brandrup J., Immergut E., Grulke E. A., Abe A., Bloch D. R.: Polymer handbook. Wiley, New York (1999).

[21] Tadokoro H., Kobayashi M., Ukita M., Yasufuku K., Murahashi S., Torii T.: Normal vibrations of the polymer molecules of helical conformation. V. Isotactic polypropylene and its deuteroderivatives. The Journal of Chemical Physics, 42, 1432-1449 (1965).

DOI: $10.1063 / 1.1696134$

[22] Pigeon M., Prud'homme R. E., Pezolet M.: Characterization of molecular orientation in polyethylene by Raman spectroscopy. Macromolecules, 24, 5687-5694 (1991).

DOI: $10.1021 / \mathrm{ma} 00020 \mathrm{a} 032$

[23] Richard-Lacroix M., Pellerin C.: Novel method for quantifying molecular orientation by polarized Raman spectroscopy: A comparative simulations study. Applied Spectroscopy, 67, 409-419 (2013).

DOI: $10.1366 / 12-06879$

[24] Richard-Lacroix M., Pellerin C.: Accurate new method for molecular orientation quantification using polarized Raman spectroscopy. Macromolecules, 46, 5561-5569 (2013). DOI: $10.1021 / \mathrm{ma} 400955 \mathrm{u}$

[25] Bower D. I.: Orientation distribution functions for uniaxially oriented polymers. Journal of Polymer Science Polymer Physics Edition, 19, 93-107 (1981).

DOI: $10.1002 /$ pol.1981.180190108

[26] Nitta K-H., Takayanagi M.: Direct observation of the deformation of isolated huge spherulites in isotactic polypropylene. Journal of Materials Science, 38, 4889-4894 (2003).

DOI: 10.1023/B:JMSC.0000004410.56145.f1

[27] Nitta K-H.: A molecular theory of stress-strain relationship of spherulitic materials. Computational and Theoretical Polymer Science, 9, 19-26 (1999).

DOI: 10.1016/S1089-3156(98)00047-6

[28] Xiong B., Lame O., Chenal J-M., Rochas C., Seguela R., Vigier G.: In-situ SAXS study of the mesoscale deformation of polyethylene in the pre-yield strain domain: Influence of microstructure and temperature. Polymer, 55, 1223-1227 (2014).

DOI: $10.1016 /$ j.polymer.2014.02.004

[29] Song Y., Nitta K-H., Nemoto N.: Deformation mechanisms of polymer thin films by simultaneous kinetic measurement of microscopic infrared dichroism and macroscopic stress. 2. Molecular orientation during necking process of isotactic polypropylene. Macromolecules, 36, 1955-1961 (2003). DOI: $10.1021 / \mathrm{ma} 0214254$ 
[30] Wang X., Michielsen S.: Isotactic polypropylene morphology-Raman spectra correlations. Journal of Applied Polymer Science, 82, 1330-1338 (2001).

DOI: $10.1002 / a p p .1968$

[31] Takayanagi M., Nitta K-H., Kojima O.: Application of lamellar clustering theory to isotactic polypropylene and direct observation of lamellar cluster morphology by electron microscopy. Journal of Macromolecular Science Part B: Physics, 42, 1049-1059 (2003).

DOI: $10.1081 / \mathrm{MB}-120023557$

[32] Nitta K-H., Takanayagi M.: Novel proposal of lamellar clustering process for elucidation of tensile yield behavior of linear polyethylenes. Journal of Macromolecular Science Part B: Physics, 42, 107-126 (2003). DOI: $10.1081 / \mathrm{MB}-120015754$
[33] Kuriyagawa M., Nitta K-H.: Structural explanation on natural draw ratio of metallocene-catalyzed high density polyethylene. Polymer, 52, 3469-3477 (2011). DOI: $10.1016 /$ j.polymer.2011.05.028

[34] Lee W. B., Wu S. Z., Song M. S.: Characterization of the orientation structure and distribution in rolled polypropylene. Journal of Materials Engineering and Performance, 5, 637-645 (1996). DOI: $10.1007 / \mathrm{BF} 02646094$

[35] Lafrance C-P., Prud'homme R. E: Characterization of the molecular orientation in highly oriented rolled polypropylene sheets by X-ray diffraction. Polymer, 35, 3927-3935 (1994).

DOI: $10.1016 / 0032-3861(94) 90277-1$ 